\title{
Thermal and Chemical Aspect in Equation of State and Relation with Generalized Thermodynamic Entropy
}

\author{
P. Palazzo \\ Sapienza University, Rome, Italy \\ E-mail: ${ }^{1}$ pierfrancesco.palazzo@uniroma1.it
}

Received 24 January 2018, Revised 5 February 2018, Accepted 17 February 2018

\begin{abstract}
First intent of the present research is to prove the rationale behind a generalized definition of thermodynamic entropy as a state and additive property inherent to any system, large or small, in any state, equilibrium or non-equilibrium. The second objective is to extend the canonical equation of state in the perspective of thermal and chemical aspect of microscopic configurations of a system related to inter-particle kinetic energy and inter-particle potential energy determining macroscopic parameters. As a consequence, a generalized state equation is formulated accounting for thermal, chemical and mechanical thermodynamic potentials characterizing any system in any state.
\end{abstract}

Keywords: Many-particle system; few-particle system; first law; second law; generalized thermodynamic entropy; generalized exergy; generalized state equation; equilibrium; non-equilibrium.

\section{Introduction}

The literature reports a definition of entropy property, proved by Gyftopoulos and Beretta, derived by replacing the heat interaction $Q$, appearing in Clausius canonical formulation, with the difference between energy $E$ and available energy $\Omega^{R}$ of a system A interacting with an external reference system, or reservoir, $\mathrm{R}$, times a parameter $1 / C_{R}$ characteristic of the reservoir:

$S_{1}-S_{0}=\frac{1}{C_{R}}\left[\left(E_{1}-E_{0}\right)-\left(\Omega_{1}^{R}-\Omega_{0}^{R}\right)\right]$

where it is proved that $C_{\mathrm{R}}=T_{\mathrm{R}}$ and $T_{\mathrm{R}}$ is the constant temperature of $\mathrm{R}$ [1]. The physical meaning of this expression is that entropy variation is determined by the amount of non-useful heat released by the system, along whatever process between initial and final states, to the reservoir at $T_{\mathrm{R}}$. Indeed, energy minus the available energy results in the non-available energy. The available energy is defined with respect to the reservoir $\mathrm{R}$ and hence corresponds to the exergy $E X^{\mathrm{R}}$, in turn depending on a fixed reference thermodynamic state, both being additive state properties. Therefore, the above expression of entropy can be turned into the following one:

$S_{1}-S_{0}=\frac{1}{T_{R}}\left[\left(E_{1}-E_{0}\right)-\left(E X_{1}^{R}-E X_{0}^{R}\right)\right]$

Considering that exergy is defined as the maximum net useful work withdrawable from a system-reservoir composite, here the physical meaning is that entropy corresponds to the minimum net non-useful heat released by the system to the reservoir at $T_{\mathrm{R}}$. As anticipated, the consequence is that the concept of heat interaction $Q$ is not more used in this definition that, instead, implies entropy being an inherent property of matter, hence it does not depend on whatever external reference system or reservoir is assumed $[2,3,4]$. The role of the reservoir is therefore auxiliary only and recent studies demonstrate that entropy can be defined with no use of the reservoir concept [5].

To corroborate this conception, the theory underpinning exergy and the entropy-exergy relationship has been elaborated also by Gaggioli who has authored an overarching treatise of all fundamental aspects of the methodology based on this property [6-9]. Significant concepts, among those demonstrated by Gaggioli, are equivalence and interconvertibility of heat and work interactions $[8,9]$. The equivalence implies the interconvertibility among interactions occurring between system and the reservoir in the sense that the thermodynamic efficiency of the conversion processes heat-to-work or mass-to-work is equal to the opposite conversion process work-to-heat or work-tomass. The interconvertibility also derives from the assumption that available energy of a system-reservoir composite is a unique state property regardless the specific process undergone from initial state to stable equilibrium state of the composite itself.

The equivalence and interconvertibility above mentioned should be extended to characterize mass and work interactions too. Mass interaction is here intended as the amount of chemical constituents mole input and or output through the control volume of the system determining variations of the inter-particle potential energy, or chemical internal energy of the system. Then, the concepts of equivalence and interconvertibility allow to infer that the definition of exergy, consisting of the maximum net useful work withdrawable from a system-reservoir composite, can be considered in its symmetric meaning as the maximum net useful heat or mass that can be extracted from the same system in the identical thermodynamic state with respect to the constant conditions of the reservoir. In other terms, the statement of equivalence stating that "work is not better than heat" [7-9] should be extended as work is not better than mass, or, more in general, one could assess that work interaction is not better than heat or mass interaction. These 
statements are the premise to think of the definition of mechanical exergy $E X^{\mathrm{M}}$ as a quantifier of the amount of maximum net useful heat or maximum net useful mass resulting from the opposite processes producing the maximum net useful work, this latter representing the canonical definition of exergy reported in the literature. That said, on the basis of the twofold relation of equivalence and inter-convertibility, a duplication of the mentioned relation to heat and mass interactions [6,7] can be equally formalized by the expressions of mechanical exergy $E X^{\mathrm{M}}$ adopting the symbolism in [1]

$$
E X^{T}=\left(W_{10}^{A R \rightarrow}\right)^{M A X}=\left(E X_{1}^{R}-E X_{0}^{R}\right)^{T}
$$

Thermal Exergy corresponding to the maximum net useful work due to thermal-energy-to-work conversion process, implying the minimum non-useful heat released to the reservoir;

$$
E X^{C}=\left(W_{10}^{A R \rightarrow}\right)^{M A X}=\left(E X_{1}^{R}-E X_{0}^{R}\right)^{C}
$$

Chemical Exergy corresponding to the maximum net useful work due to chemical-energy-to-work conversion process, implying the minimum non-useful mass released to the reservoir

$$
E X^{M}=\left(Q_{10}^{A R \rightarrow}\right)^{M A X}=\left(E X_{1}^{R}-E X_{0}^{R}\right)^{M}
$$

Mechanical Exergy corresponding to the maximum net useful heat due to mechanical-energy-to-heat conversion process implying the minimum non-useful work released to the reservoir;

$$
E X^{M}=\left(M_{10}^{A R \rightarrow}\right)^{M A X}=\left(E X_{1}^{R}-E X_{0}^{R}\right)^{M}
$$

Mechanical exergy corresponding to the maximum net useful mass due to mechanical-energy-to-mass conversion process implying the minimum non-useful work released to the reservoir.

The above definitions, considering that exergy is a nonconservative additive state property, lead to formulate the generalized exergy [6,7]:

$$
E X^{G}=E X^{T}+E X^{C}+E X^{M}
$$

that can be regarded as the maximum net useful (heat, mass or work) interaction between system and the reservoir that represents the reference system determining the exergy content of the system thermodynamic state. The physical meaning of generalized exergy lies in the equivalence and interconvertibility characteristic among all interactions occurring in whatever process, reversible or irreversible, thus requiring that all involved interactions be evaluated in terms of exergy property. Finally, the generalized exergy accounts for each and every kind of interaction involved in the process from any thermodynamic state to the stable equilibrium state. Considering the relationship between exergy and entropy, as expressed by the equation discussed so far, it is a logical consequence to derive an alternative formulation of entropy from exergy on the basis of previous definitions. Nevertheless, entropy definition can be generalized to account for all contributions of internal energy deriving from temperature (inter-particle kinetic energy), potential (interparticle potential energy) and pressure (determined by both kinetic energy and potential energy) in a perspective of microscopic description of statistical physics correlated to the macroscopic phenomena and properties. Hence, a generalized entropy $S^{G}$ definition can be stated as the result of thermal $S^{T}$, chemical $S^{C}$ and mechanical $S^{M}$ reversible contributions and irreversible productions [11]:

$$
S^{G}=S^{T}+S^{C}+S^{M}
$$

\section{Generalized Thermodynamic Entropy Definition}

Gibbs relation expressing the First Law for a system composed by 1 mole of $r$ chemical constituents and the volume $V$ as the only parameter that confines the system (control mass and control volume), is:

$d U=T d S+\mu_{1} d n_{1}+\ldots .+\mu_{r} d n_{r}-P d V$

from which (thermal) entropy, associated to thermal internal energy, with units of measure in $J /($ mole $K)$ is obtained, expressed in differential terms, as follows:

$d S^{T}=\left(d U-\mu_{1} d n_{1}-\ldots-\mu_{r} d n_{r}+P d V\right) / T$

$=\frac{d U}{T}-\frac{\mu_{1} d n_{1}}{T}-\ldots .-\frac{\mu_{r} d n_{r}}{T}+\frac{P d V}{T}$

The additivity of generalized thermodynamic entropy property in Eq. (8), implying the balance of all contributions, underpins its derivation based on Gibbs relation in Eq. (9) from the combination of thermal, chemical and mechanical components of internal energy, referred to as $U^{T}, U^{C}$ and $U^{M}$ respectively. Two forms can be used to express the generalized thermodynamic entropy property. The first form considers the temperature as an integrating factor $[12,13]$ :

$$
\begin{aligned}
& d S^{G}=d S^{T}+d S^{C}+d S^{M} \\
& =\frac{d U}{T}=\frac{d\left(U^{T}+U^{C}+U^{M}\right)}{T} \\
& =d S^{T}+\frac{\mu_{1} d n_{1}}{T}+\ldots .+\frac{\mu_{r} d n_{r}}{T}+\frac{P d V}{T}
\end{aligned}
$$

The second form considers the chemical potential as an equivalent integrating factor:

$$
\begin{aligned}
& d S^{G}=d S^{T}+d S^{C}+d S^{M} \\
& =\frac{d U}{\mu}=\frac{d\left(U^{T}+U^{C}+U^{M}\right)}{\mu} \\
& =\frac{T d S}{\mu}+\frac{\mu_{1} d n_{1}}{\mu}+\ldots .+\frac{\mu_{r} d n_{r}}{\mu}+\frac{P d V}{\mu}
\end{aligned}
$$

Hence, entropy derived from Gibbs relation is associated to all thermal, chemical and mechanical interactions occurring in closed and or open systems.

On the other side, an adiabatic reversible process, identified by definition as isoentropic because experiencing work interaction only (no heat or mass interaction occur) would imply that no entropy variation occurs: hence it seems to be in contradiction with the conclusion that Gibbs relation is based on a definition of entropy accounting for all forms of energy and interactions. An isoentropic process requires that entropy in Gibbs relation should not be considered as a total entropy: in fact, it is an entropy contribution associated to thermal and or chemical internal energy and heat and or mass interaction only and for this very reason is not associated to mechanical energy and work interaction. While the generalized definition of entropy, derived from Gibbs 
relation itself, accounts for all contributions. The isoentropic process therefore displays an incoherence. Moreover, an adiabatic reversible process cannot be isoentropic, again as a consequence of Gibbs relation, thus it has to be characterized by a change in mechanical entropy $S^{M}$ constituting a contribution, in addition to thermal entropy $S^{T}$ and chemical entropy $S^{C}$, implicitly accounted for in the Gibbs relation. This apparent contradiction, is resolved considering that the generalized definition of entropy, in two forms of Eqs. (11) and (12), is extended to encompass thermal entropy, chemical entropy and mechanical entropy contributions related to thermal, chemical and mechanical internal energy components associated to heat, mass and work interactions with the reference external system or reservoir $R$. According to this rationale, a definition of mechanical entropy and chemical entropy has been proposed $[11,12]$ under the formulation previously established for the expression of thermal entropy in Eqs. (1) and (2), as reported in the literature [1-4]. On this basis, it would be also possible to extend the concept of adiabatic availability [1] by addressing to "non-interaction availability" in the sense that no heat, no mass and no work interaction occur between system and reservoir, or among different portions of the system itself.

The differential form of the definition of thermal entropy $S^{T}$, referred to 1 mole of any chemical constituent or set of chemical constituents of the system, can be expressed as:

$d S^{T}=\frac{\delta Q}{T}=\frac{d E_{R}^{T}}{T_{R}}=\frac{d\left(E^{T}-E X^{T}\right)}{T_{R}}$

where the term $1 / T$ constitutes the integration factor which makes the elemental heat interaction $\delta Q$ an exact differential according to Pfaff theorem $[12,13] . T_{R}$ is the constant temperature of the reservoir $\mathrm{R}$.

Contributions of entropy components resulting from Gibbs relation, as above demonstrated, imply the equivalence of State equation specially expressed for all forms of internal energy.

\section{Mechanical-Thermal State Equation}

The canonical mechanical-thermal State equation results from the equivalence between inter-particle kinetic energy associated to the temperature depending on particles relative velocity, and the pressure generated by particles kinematic configuration, that is:

$P V=n \bar{R} T$

where $\bar{R}=\frac{J}{\mathrm{~mol} \cdot \mathrm{K}}$ is the universal constant. The equivalence of units of measure of this State equation is analyzed resulting in $J=\mathrm{mol} \cdot \frac{\mathrm{mol} \cdot \mathrm{K}}{\mathrm{m}} \cdot \mathrm{K}=J$ from which $J=J$ that is identically verified. The physical meaning, in the framework of statistical physics and the Kinetic Theory, is that the content of macroscopic mechanical internal energy of a system as a whole is equivalent to the content of its thermal internal energy determined by microscopic inter-particle kinetic energy of the constituting particles of whatever system, as assumed by the thermal-mechanical State equation.

Vice versa, if pressure, associated to the density depending on particles collision frequency, is considered, then a thermal-mechanical state equation should appear as:
$T S^{T}=\frac{P V}{n \bar{R}} S^{M}$

Units of measure of this equation are $K \cdot \frac{J}{\mathrm{~mol} \cdot \mathrm{K}}=\frac{1}{\mathrm{~mol}} \cdot \frac{\mathrm{mol} \cdot \mathrm{K}}{J} \cdot \mathrm{J} \cdot \frac{\mathrm{J}}{\mathrm{mol} \cdot \mathrm{K}}$ from which $\frac{\mathrm{J}}{\mathrm{mol}}=\frac{J}{\mathrm{~mol}}$ that is verified as in the previous equivalence. The equivalence of units of measure of both members of the two State equations are therefore congruent. This fact proves that, at microscopic level, both temperature and pressure result from the kinetic energy of particles interacting each other and interacting with the system confinement wall.

\section{Mechanical-Chemical State Equation}

The differential form of the definition of chemical entropy $\mathrm{S}^{\mathrm{C}}$, referred to one mole of any chemical constituent or set of chemical constituents of the system, can be expressed as:

$d S^{C}=\frac{\delta M}{\mu}=\frac{d E_{R}^{C}}{\mu_{R}}=\frac{d\left(E^{C}-E X^{C}\right)}{\mu_{R}}$

The term $1 / \mu$ constitutes the integrating factor which makes the elemental mass interaction $\delta M$ an exact differential again according to Pfaff theorem [12,13]. This results from the fact that chemical entropy, as thermal entropy, is an inherent property of any system, in any state.

On the other hand, a mechanical-chemical State equation can be envisaged resulting from the equivalence between inter-particle potential energy, associated to the potential depending on particles relative position, and the pressure generated by particles geometric configuration, associated to the density depending on particles relative position, and can be expressed as:

$P V=n \bar{R} \mu$

In this case, considering that the unit of measure of the chemical potential $\mu$ is $J / m o l$ the units of measure equivalence implies that:

i) from mechanical-chemical State equation $P V=n \bar{R} \mu$ then $J=\mathrm{mol} \cdot \frac{\mathrm{J}}{\mathrm{mol} \cdot \mathrm{K}} \cdot \frac{\mathrm{J}}{\mathrm{mol}} \Rightarrow 1=\frac{\mathrm{J}}{\mathrm{mol} \cdot \mathrm{K}} \quad$ corresponding to $J=m o l \cdot K$;

ii) from $P V=n \bar{R} \mu$, then $\bar{R}=\frac{P V}{n T}$ so that units of measure equivalence $\bar{R}=\frac{J}{\mathrm{~mol} \cdot \mathrm{K}} \quad$ using the $\quad J=\mathrm{mol} \cdot \mathrm{K}$, becomes $\bar{R}=\frac{J}{J}$ and, by virtue of mechanical-thermal State equation, then $J=\mathrm{mol} \cdot \frac{J}{J} \cdot K=\mathrm{mol} \cdot K=J \Rightarrow J=J$

iii) substituting $\bar{R}=\frac{J}{J}$ again in the mechanical-chemical State equation $P V=n \bar{R} \mu$ then $J=m o l \cdot \frac{J}{J} \cdot \frac{J}{\mathrm{~mol}}$ from which $J=J$ that is identically verified also in this case and hence confirms the physical equivalence between mechanical internal energy and the content of its chemical internal energy determined by inter-particle potential energy assumed by the mechanical-chemical state equation. 
Similarly, to the previous case of the mechanical-thermal state equation, here the physical meaning is adopted again from the framework of statistical physics and the kinetic theory. Hence, the content of macroscopic mechanical internal energy of a system as a whole is equivalent to the content of its chemical internal energy determined by microscopic inter-particle potential energy of the constituting particles of the same system, as assumed by the mechanical-chemical state equation.

The above points i), ii) and iii) include equations that are to be intended as units of measure equivalences only and are not numerical equalities. Those equations are equalities in case proper values are assigned to all properties and parameters. In particular, $\bar{R}=k_{B} N_{A}$ where $k_{B}$ is the Boltzmann constant and $N_{A}$ is the Avogadro number. Thus $k_{B}=\frac{\bar{R}}{N_{A}} \quad$ so that, in terms of units of measure, $k_{B}=\frac{J}{\mathrm{~mol} \cdot \mathrm{K}} \cdot \frac{\mathrm{mol}}{\text { molecules }}=\frac{J}{\text { molecules } \cdot K}$. Therefore, being $\bar{R}=\frac{J}{\mathrm{~mol} \cdot \mathrm{K}} \quad$ and $\quad k_{B}=\frac{J}{\text { molecules } \cdot K}$, then the following equivalences apply $\quad J=\bar{R} \cdot \mathrm{mol} \cdot K \quad$ and $J=k_{B} \cdot$ molecules $\cdot K$; hence, the combination of the two equivalences

$$
J=\operatorname{mol} \frac{J}{\operatorname{molK}} K=\text { molecules } \cdot \frac{J}{\text { molecules } \cdot K} \cdot K=J
$$

proves that $\bar{R}$ and $k_{B}$ are equivalent in terms of units of measure and, more precisely, are dimensionless, whereas they have different numerical values. The consequence is that, as $\bar{R}$ and $k_{B}$ satisfy the units of measure equivalence, then $J=m o l \cdot K$ is true for the specific case referred to one mole and $J=$ molecules $K$ is in turn true for the general case of all molecules constituting any system at microscopic level.

Also in this case, if pressure, associated to the density depending on particles collision intensity, is considered, then a chemical-mechanical State equation should become:

$$
\mu S^{C}=\frac{P V}{n \bar{R}} S^{M}
$$

Again, the equivalence of units of measure is $\frac{J}{\mathrm{~mol}} \cdot \frac{\mathrm{J}}{\mathrm{mol} \cdot \mathrm{K}}=\frac{1}{\mathrm{~mol}} \cdot \frac{\mathrm{mol} \cdot \mathrm{K}}{\mathrm{J}} \cdot \mathrm{J} \cdot \frac{\mathrm{J}}{\mathrm{mol} \cdot \mathrm{K}} \quad$ from $\quad$ which $\frac{J}{m o l}=\frac{J}{m o l}$ that is identically verified. This fact again proves that, at microscopic level, both potential and pressure result from the potential energy of particles interacting each other.

Considering that, from the set of expressions of state equation, $T=\frac{P V}{n \bar{R}}$ and $\mu=\frac{P V}{n \bar{R}}$, then the unit of measure equivalence of $T=\mu$, expressed as $K=\frac{J}{m o l}$ is proved.

It is noteworthy that, in terms of dimensional analysis of properties, and equivalence of units of measure, considering that $J=\mathrm{mol} \cdot \mathrm{K}$, the thermal entropy is $\frac{J}{\mathrm{~mol} \cdot \mathrm{K}}=1$ and the chemical entropy is $\frac{\mathrm{J} / \mathrm{mol}}{\mathrm{J} / \mathrm{mol}}=1$, thus, the two properties are dimensionless. From physical point of view this result confirms that entropy represents an indicator of the subdivision, among all available degrees of freedom, of whatever physical quantity defining the state or process of a system, no matter the nature of quantity and system under consideration. In different terms, "Entropy is the ratio between macro (multi-particle) energy and a representative particle energy, thus dimensionless".

\section{Generalized State Equation}

As regard the differential form of mechanical entropy $S^{M}$, appearing as a contribution in Gibbs equation:

$$
\begin{aligned}
& d S^{G}=\frac{d U}{P V}=d S^{T}+d S^{C}+d S^{M} \\
& =\frac{T d S}{P V}+\frac{\mu_{1} d n_{1}}{P V}+\ldots .+\frac{\mu_{r} d n_{r}}{P V}+\frac{P d V}{P V}
\end{aligned}
$$

the following expression has been proposed $[11,12]$ :

$$
d S^{M}=\frac{\delta W}{P V}=\frac{d E_{R}^{M}}{P_{R} V_{R}}=\frac{d\left(E^{M}-E X^{M}\right)}{P_{R} V_{R}}
$$

where the term $1 / P V$ in turn constitutes the integrating factor which makes the work interaction an exact differential according to Pfaff theorem $[12,13]$.

Considering both thermal-mechanical and chemicalmechanical contributions, their combination becomes $T S^{T}+\mu S^{C}=\frac{P V}{n \bar{R}} S^{M} \quad$ from which the following Generalized State equation is derived:

$$
P V=\frac{n \bar{R}}{S^{M}}\left(T S^{T}+\mu S^{C}\right)
$$

Coming back to the initial discussion on internal energy, the terms representing finite contributions (per mole) can be written as thermal internal energy $U(T)=T S^{T}$, chemical internal energy $U(\mu)=\mu S^{C}$ and mechanical internal energy $U(P)=\frac{P V}{n \bar{R}} S^{M}$. As above reported, $T=\frac{P V}{n \bar{R}}$ and $\mu=\frac{P V}{n \bar{R}}$ so that:

$$
\begin{aligned}
& U=U(T, \mu, P)=U(T)+U(\mu)+U(P) \\
& =T S^{T}+\mu S^{C}+\frac{P V}{n \bar{R}} S^{M} \\
& =\frac{P V}{n \bar{R}} S^{T}+\frac{P V}{n \bar{R}} S^{C}+\frac{P V}{n \bar{R}} S^{M} \\
& U=\frac{P V}{n \bar{R}}\left(S^{T}+S^{C}+S^{M}\right)=\frac{P V}{n \bar{R}} S^{G}=P^{G} S^{G}
\end{aligned}
$$

where $P^{G}$ represents the generalized thermodynamic potential and $S^{G}$ the generalized thermodynamic entropy. Then the equivalence of all forms of internal energy is established and, on this basis, the sum of thermal, chemical and mechanical entropies can be defined as generalized thermodynamic entropy [11,12]: $S^{G}=S^{T}+S^{C}+S^{M}$.

Hence, the generalized state equation can be also expressed in the following form: 


$$
P V=n \bar{R} P^{G}
$$

The dimensional equivalence of units of measure is $\frac{J}{\mathrm{~mol}}=\frac{J}{\mathrm{~mol}} \cdot \frac{\mathrm{mol} \cdot \mathrm{K}}{J}=1$ that results identically verified.

The canonical definition of thermal entropy addresses to the degree of distribution of kinetic energy among the particles constituting the system: "Entropy as defined by Quantum Thermodynamics as a measure of the distribution of a system's internal energy at any given instant of time amongst the available internal degrees of freedom, i.e., the energy eigenlevels of the system" [14]. For this very reason, thermal entropy $S^{T}$ can be regarded as a kinematic entropy addressing to the relative velocity microscopic configuration. Similarly, the definition of chemical entropy $S^{C}$ is based on the distribution of potential energy among the particles constituting the system hence the definition of geometric entropy seems addressing to the relative position microscopic configuration. Finally, the mechanical entropy $S^{M}$ accounts for density of energy depending on the volume of the system considered as the only parameter governing work interaction moving the (gravitational or electromagnetic) weight process of the external system [1]. Volume determines frequency of collisions in the case of kinetic energy generating the work of kinetic origin, and determines intensity of interactions in the case of potential energy generating the work of potential origin.

Finally, using the same formulation of entropy adopted for thermal, chemical and mechanical contributions, a definition of Generalized Thermodynamic Entropy is the following:

$$
d S^{G}=\frac{d\left(E^{G}-E X^{G}\right)}{P^{G}}
$$

\section{Conclusions}

The definition of Generalized Thermodynamic Entropy, resulting from thermal, chemical and mechanical contributions and production processes, is the property accounted for extending the canonical state equation considering both thermal or chemical aspects of entropyexergy relationship and the dependency between microscopic state and macroscopic parameters and properties of any system in any state. The physical meaning of State equation is assumed deriving from statistical physics and the microscopic configuration of any system correlated to the macroscopic thermodynamic state. Consequently, thermal-mechanical and chemical-mechanical aspects of state equation have been analyzed and specific direct and inverse formulations have been proposed as the components of a generalized state equation. Hence, the formulation of the generalized state equation is derived accounting for all forms of energy and entropy properties characterizing any thermodynamic state of a system.

Outcomes of the present research can be regarded as a theoretical paradigm highlighting an extended perspective on studies already published $[18,19]$ on the state equation of real systems and their properties along irreversible processes among non-equilibrium states.

\section{Nomenclature}

$A$ : internal system

$C$ : constant parameter
$E$ : energy $J / m o l$

$E X^{G}$ : generalized exergy $\mathrm{J} / \mathrm{mol}$

$E X^{C}$ : chemical exergy $\mathrm{J} / \mathrm{mol}$

$E X^{M}$ : mechanical exergy $\mathrm{J} / \mathrm{mol}$

$E X^{T}$ : thermal exergy $\mathrm{J} / \mathrm{mol}$

$k_{B}$ : Boltzmann constant $\mathrm{J} /$ molecules $\cdot \mathrm{K}$

$M$ : mass interaction $\mathrm{J} / \mathrm{mol}$

$n$ : mole $\mathrm{mol}$

$P$ : pressure $P a$

$P^{G}$ : generalized potential $\mathrm{J} / \mathrm{mol}$

$Q$ : heat interaction $J / m o l$

$R:$ reservoir

$\bar{R}$ : universal constant $\mathrm{J} / \mathrm{mol} \cdot \mathrm{K}$

$S^{C}$ : chemical entropy $\mathrm{J} / \mathrm{mol} \cdot \mathrm{K}$

$S^{G}$ : generalized entropy $\mathrm{J} / \mathrm{mol} \cdot \mathrm{K}$

$S^{M}$ : mechanical entropy $\mathrm{J} / \mathrm{mol} \cdot \mathrm{K}$

$S^{T}$ : thermal entropy $\mathrm{J} / \mathrm{mol} \cdot \mathrm{K}$

$T$ : temperature $\mathrm{Pa}$

$U$ : internal energy $\mathrm{J} / \mathrm{mol}$

$V$ : volume $\mathrm{m}^{3}$

$W$ : work interaction $J / m o l$

$\mu$ : chemical potential $J / \mathrm{mol}$

$\Omega$ : available energy $J / \mathrm{mol}$

\section{References:}

[1] E.P. Gyftopoulos, G.P. Beretta, Thermodynamics: Foundations and Applications, Dover Publication: New York 2005.

[2] E.P. Gyftopoulos, "Entropy: An Inherent, Non-statistical Property of any System in any State," Int. J. of Thermodynamics, 3, 107-115, 2006.

[3] G.P. Beretta, "Axiomatic Definition of Entropy for Nonequilibrium States," Int. J. of Thermodynamics, 11, $39-48,2008$.

[4] E. Zanchini, G.P. Beretta, "Removing Heat and Conceptual Loops from the Definition of Entropy," Int. J. of Thermodynamics, 13, 67-76, 2008.

[5] E. Zanchini, G.P. Beretta, "A Definition of Thermodynamic Entropy Valid for Non-equilibrium States and Few-particle Systems," arXiv 2014;1411.5395

[6] W.R. Dunbar, N. Lior and R.A. Gaggioli "The Component Equations of Energy and Exergy," J. of Energy Resources Technology, 114, 1992

[7] R.A. Gaggioli “Available Energy and Exergy," Int. J. of Applied Thermodynamics, 1, 1-8, 1992

[8] R.A. Gaggioli, D.H. Richardson, "Available Energy Part I: Gibbs Revisited," J. Energy Resources Technology, 124, 105-109, 2002

[9] R.A. Gaggioli, D.M. Paulus, "Available Energy - Part II: Gibbs Extended," J. Energy Resources Technology, 124, 110-115, 2002 
[10] P. Palazzo, "Theorem of Necessity and Sufficiency of Stable Equilibrium for Generalized Potential Equality between System and Reservoir," J. of Modern Physics, 5, 2003-2011, 2014.

[11] P. Palazzo, "A Method to Derive the Definition of Generalized Entropy from Generalized Exergy for Any State in Many-Particle Systems," Entropy, 17,20252038, 2015.

[12] P. Palazzo, "A Generalized Statement of HighestEntropy Principle for Stable Equilibrium and NonEquilibrium in Many-Particle Systems," J. Modern Physics, 7, 344-357, 2016

[13] L. Pogliani, M.N. Berberan-Santos, "Constantin Carathéodori and the Axiomatic Thermodynamics," $J$. Mathemat Chem., 28, 1-3, 2000

[14] M. von Spakovsky, "Comparison of the Nonequilibrium Predictions of Quantum Thermodynamics at the Atomistic Level with Experimental Evidence," IMECE Conference, 2011
[15] S.J. Kline, The Low-Down on Entropy and Interpretive Thermodynamics, DCW Industries, 1999.

[16] P. Atkins, J. de Paula, Physical Chemistry, New York, DCW Industries, 1999.

[17] T.L. Hill, Statistical Mechanics. Principles and Selected Applications, Dover, New York, 1987.

[18] R.F. Checoni, S.P. Ravagnani, "Studies about an Equation of State for Pure Associated Fluids: Temperature Dependent Co-Volume Accounting a Physically Consistent Repulsive Term," Int. J. Thermodynamics, 16, 20-27, 2013.

[19] R.F. Checoni, M. Aznar, "Comparative Study between Cubic and Non-Cubic Equations of State Using Carnahan-Starling Repulsive Term: Application of Temperature-Dependent Alpha and Beta Functions," Int. J. Thermodynamics, 17, 21-26, 2014. 